\title{
How Network-based and Set-based Visualizations Aid Consistency Checking in Ontologies
}

\author{
Yuri Sato \\ Visual Modelling Group, School of \\ Computing, Engineering and \\ Mathematics, University of Brighton \\ y.sato@brighton.ac.uk
}

\author{
Gem Stapleton \\ Visual Modelling Group, School of \\ Computing, Engineering and \\ Mathematics, University of Brighton \\ g.e.stapleton@brighton.ac.uk
}

\author{
Mateja Jamnik \\ Computer Laboratory, Faculty of \\ Computer Science and Technology, \\ University of Cambridge \\ mateja.jamnik@cl.cam.ac.uk
}

\author{
Zohreh Shams \\ Computer Laboratory, Faculty of \\ Computer Science and Technology, \\ University of Cambridge \\ zohreh.shams@cl.cam.ac.uk
}

\begin{abstract}
Ontologies describe complex world knowledge in that they consist of hierarchical relations, such as $i s-a$, which can be expressed by quantifiers or sets, and various binary relations, which can be expressed by links or networks. Should hierarchical relations be distinguished from other binary relations as essentially different ones in building cognitively accessible systems of ontologies? In this study, two kinds of ontology visualizations, a network-based visualization (SOVA) and a set-based visualization (concept diagrams), are empirically compared in the case of consistency checking. Participants were presented with one diagram and then asked to answer the question of whether the meaning of the diagram was contradictory. Our results showed that SOVA is more effective than concept diagrams, suggesting that to represent hierarchical and binary relations of ontologies in a way based on networks suits human cognition when checking ontologies' consistencies.
\end{abstract}

\section{CCS CONCEPTS}

-Human-centered computing $\rightarrow$ Visualization theory, concepts and paradigms; Empirical studies in visualization; Information visualization;

\section{KEYWORDS}

semantic network, concept diagram, ontologies, consistency, cognitive science

\section{ACM Reference format:}

Yuri Sato, Gem Stapleton, Mateja Jamnik, Zohreh Shams, and Andrew Blake. 2017. How Network-based and Set-based Visualizations Aid Consistency Checking in Ontologies. In Proceedings of VINCI '17, Bangkok, Thailand, August 14-16, 2017, 5 pages.

DOI: http://dx.doi.org/10.1145/3105971.3105988

Permission to make digital or hard copies of all or part of this work for personal or classroom use is granted without fee provided that copies are not made or distributed for profit or c ommercial a dvantage a nd that c opies b ear t his n otice a nd the full citation on the first $\mathrm{p}$ a ge. C opyrights f or components of $\mathrm{t}$ his $\mathrm{w}$ ork o wned b y others than ACM must be honored. Abstracting with credit is permitted. To c opy otherwise, or republish, to post on servers or to redistribute to lists, requires prior specific permission and/or a fee. Request permissions from permissions@acm.org. VINCI '17, Bangkok, Thailand

(c) 2017 ACM. 978-1-4503-5292-5...\$15.00

DOI: http://dx.doi.org/10.1145/3105971.3105988
Andrew Blake

Visual Modelling Group, School of Computing, Engineering and

Mathematics, University of Brighton a.l.blake@brighton.ac.uk

(i)

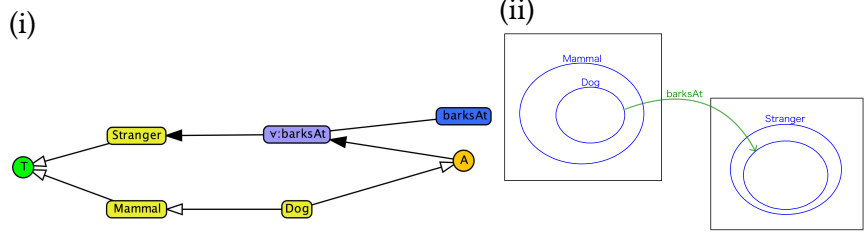

Figure 1: (i) Network-based visualization "SOVA" and (ii) Set-based visualization "Concept Diagram" for Each dog is a mammal and All dogs bark at only strangers.

\section{INTRODUCTION}

The qualities of our thinking, reasoning and judgement depend on whether we can efficiently use world knowledge, which is often described by an ontology. In general, ontologies are not limited to simple ones such as taxonomies, containing hierarchical verbs of is- $a$ or part-of relations, which are expressed by unary predicates; e.g., Each dog is a mammal. Rather, ontologies contain other various verb relations besides the above hierarchical verbs, which are expressed by binary predicates; e.g., All dogs bark at only strangers. In order for non-experts to access the complex knowledge captured in ontologies efficiently, how could the ontologies be represented in a mental or external way? Behind this question there is a more general one: should hierarchical relations be distinguished from binary relations as essentially different ones in building ontology?

If all relations, expressed by unary and binary predicates, are represented uniformly, they can be represented, for example, by linking concepts or objects in a network system (as we explain later, different kinds of links are needed). Figure 1(i) shows a network-based visualization of Each dog is a mammal and All dogs bark at only strangers. By contrast, if hierarchical relations are intended to be represented differently, it is natural to consider that they are expressed by set-theoretical relations such as inclusion and exclusion between sets (or individuals). We can, thus, consider a set-based visualization, as in Figure 1(ii). The two visualizations are equivalent in semantic information but differ in form.

Ontologies are typically built by using a language (e.g., OWL) in a certain environment (e.g., Protégé) (cf. [7, 21]). However, languages are used for constructing a machine readable form, and not 
because they are suitable or easy-to-understand for humans. In fact, various visualizations for ontology building have been developed $^{1}$. Given this, it is expected that visual forms other than sentential or symbolic forms can play an essential role in exploring effective representations of ontologies.

Almost all visualizations for ontologies are based on networks (node-link diagrams) (cf. [23]). Among them, SOVA network [16], as in Figure 1(i), is used in this study. In order to challenge the dominant view that the network-based representation is the most effective method, we pay attention to the recent development of diagrammatic logic. In addition to a well-known set visualization, namely, Euler diagrams, its extended system, called concept diagrams (Figure 1(ii) $[14,22,26]$ ) is analyzed. This study conducts the theoretical and empirical comparison between network-based visualizations (SOVA networks) and set-based visualizations (concept diagrams) in consistency checking.

\section{TASK ANALYSIS}

\subsection{Network-based visualization: SOVA}

Network representations for semantic information are often called semantic networks [10]. Their components are nodes and links. Typically, nodes are labelled boxes, representing concepts and individuals. Links are labelled arrows, representing relations (binary predicates) between concepts or individuals. The arrows' directions show the directionality from subjects to objects: the concepts in the source are subjects and ones in the target are objects.

Some extensions and formulations of semantic networks have been provided (e.g., KL-ONE [2], which encouraged the development of OWL). In the literature of ontology visualization, however, such extended systems of semantic networks have been rarely used. The expressive power is still limited. For example, VOWL in [19] is not expressive enough to deal with notable OWL restrictions. In contrast to the existing systems, [16] developed a kind of semantic network named "SOVA" (Simple Ontology Visualization API) in a way to overcome the expressive limitation.

One of the distinctive points of SOVA is that it has two kinds of arrows: white-headed and black-headed ones. Consider them in Figure 1(i). (1) White-headed arrows are used to express hierarchical relations. Dog is linked by a white-headed arrow to Mammal. This means that if there are dogs, all of them are mammals. Also Mammal is linked by a white-headed arrow to T (Things). This means that if there are mammals, all of them are things in the world. (2) Black-headed arrows are used to express binary relations. Here the arrows through the boxes $\forall$ :- express that the source is related to only the target. Dog is linked by a white-headed arrow to A (Anonymous sets). A is linked by a black-headed arrow to Stranger through $\forall$ :barksAt. This means all dogs are related to only strangers, under the relationship "barks at".

However, it is questionable whether use of different kinds of arrow in one system can be cognitively effective. Here the meanings of white-headed arrows and black-headed arrows are defined in an artificial or stipulative way. As arrows are distinguished purely in a syntactic way, there is no necessity for white-headed arrows to express subsumptions. In other words, there is no particular constraint on ways of corresponding syntactic objects and their

\footnotetext{
${ }^{1} \mathrm{http}: / /$ protegewiki.stanford.edu/wiki/Visualization
}

semantics. So users of the network-based visualization SOVA are required to be conscious of these semantic "rules". The relevant cognitive load could be detrimental to users' performances on interpreting and reasoning.

\subsection{Set-based visualization: concept diagram}

To avoid the potentials of effortful thinking, we can choose (or design) a representation which is matched with what it is required to express. Such line of approach has been discussed in so-called "distributed cognition" research in the literature of cognitive science. As emphasized in a seminal study of Zhang and Norman [27], well-matched representations are effective in that the internal processing of rules in interpreting and reasoning can be (partially) distributed to the physical constraints of external representations. As a representation which can contribute to the distributed cognition, we focus on a set visualization, namely, Euler diagrams.

It is assumed that hierarchical relations are naturally paraphrased by set-theoretical relations. For example, is- $a$ can be described by subsumption or set membership; the relation between sibling concepts, that is, a concept is independent of another concept, can be described by set disjointness. Such abstract relations of sets (or individuals) can be realized as the spatial relations of concrete forms such as circles, ellipses, or points. Such visualizations are called Euler diagrams and have been often used for interpreting and reasoning about categorical statements and set-theoretical relations [1]. Sato et al. [25] provide partial evidence that Euler diagrams are well-matched with set-theoretical information. They showed that the extraction of set-theoretical relations from the Euler diagrams is cognitively effortless, by comparing the time-speed for interpreting Euler diagrams and Venn diagrams.

In order to handle information contained in ontologies, we cannot continue to use such simple and natural system of Euler diagrams, as it expresses only a fragment of monadic first-order logic $[9,20]$. As an extended system for ontologies, we adopt concept diagrams [14, 22, 26]. Like a semantic network, this system uses arrows to express binary predicates other than unary predicates of hierarchical (set-theoretical) verbs ${ }^{2}$. How does this extension affect users' comprehension of the visualization system?

Consider Figure 1(ii). First, the fundamentals of Euler diagrams are that (1) the set of individuals sharing common properties are enclosed by circles, and (2) spatial-relationships between circles or points represent set relationships. The circle Dog is inside the circle Mammal. This naturally delivers the meaning that if there are dogs, all of them are mammals. Second, the solid arrow labelled barksAt connects from Dog to the unlabelled circle inside Stranger. This means that all dogs bark at only strangers. The translation that the subject term has the quantifier all is intuitive since the source of the verb arrow is touched with the whole set of dog. The diagram imposes a constraint on the possible ways in which we can interpret it. By contrast, the translation that the object term has the quantifier only is not the case. The translation of the arrow uses an artificial or stipulative rule in that the way in which we interpret is less constrained. So concept diagrams cannot be free from artificial rules of semantics. Rather, they can reduce the number of

\footnotetext{
${ }^{2}$ The view of set-based visualization is shared in the hybrid approach combining hierarchical trees (or Shneiderman's treemaps) [17]) and networks (e.g., [4, 11, 28]) It is not clear if their approach is applicable to ontologies.
} 
such rules, compared to a network-based visualization SOVA. As a result, it would be expected that concept diagrams partially realize distributed cognition, unlike SOVA networks.

\subsection{Checking ontology consistency}

We focus on a logical task of consistency checking in ontology visualizations using SOVA and concept diagrams. If there can be at least one situation in which all assertions are true at the same time, the assertions are consistent (not contradictory). On the other hand, if there cannot be a situation in which all assertions are true at the same time, the assertions are contradictory (not consistent). Consistency (and contradiction) is a crucial notion in logical reasoning. Especially in the literature on ontology engineering, to identify (and then repair) an inconsistent ontology is really needed in ontology management and maintenance (e.g., $[8,12,15])$. Currently, however, the cognitive underpinning of such logical ability has been seldom investigated experimentally. Thus, little is known about people's actual capability of consistency checking in ontologies, although there are some implementation works including user evaluation tests $[3,18]$.

The following example is one of the basic forms of inconsistencies (contradictions) in quantified assertions. Assertions (a) and (b) are contradictory: (a) If there are dwarfs, all of them are human; There is an individual " $a$ " which is both dwarf and human; and (b) There are no individuals which are both dwarf and human. In contrast, the following assertions are not contradictory: (c) If there are $d$ warfs, all of them are human. (d) There are no individuals which are both dwarf and human. The assertions say nothing about whether there are some individuals which are dwarf and/or human. So it is possible that no dwarf and/or human exists; that is, the sets can be empty. Thus, there is at least one situation in which the incoherent assertions hold at the same time.

In ontology building, people can write each axiom by using a language in an environment such as Protégé and then check the correctness by viewing automatically-generated visualizations in which information of each axiom is expressed in a merged way (i.e., as a single diagram). In this study, we focus on the above setting (i.e., using single 'merged' diagrams).

In the context of ontologies, the comprehension of various binary relations other than hierarchical verbs is needed. Thus, we can consider case (1) relevant to hierarchy but not to binary relations and case (2) relevant to hierarchy and binary relations, which are either consistent or inconsistent, as shown in Figure $2^{3}$. In Case (1), processing binary relations is not needed in checking (in)consistency. On the other hand, in Case (2), processing binary relations is needed. In Case $\left(2_{2}\right)$ of Figure 2, there is a Dock individual "a" and there is a watercraft individual which is stored in "a". This contradicts the fact that no individual is both a vehicle and a watercraft. In Case $\left(2_{1}\right)$ of Figure 2, there is a Garage individual "a" and there is a vehicle individual which is stored in "a". Regarding the existence of the vehicle individual, there are two possibilities: (i) the individual is both vehicle and watercraft and (ii) the individual is vehicle but not watercraft. If the vehicle individual is the

\footnotetext{
${ }^{3}$ We note that the SOVA images used here were based on the Protégé plug-in that generates SOVA from OWL files. However, this plug-in does not produce diagrams that involve inverse. Therefore, we manually added the standard description-logic style annotation, ${ }^{-}$, to nodes for properties that were intended to be inverses.
}

$\left(1_{1}\right)$

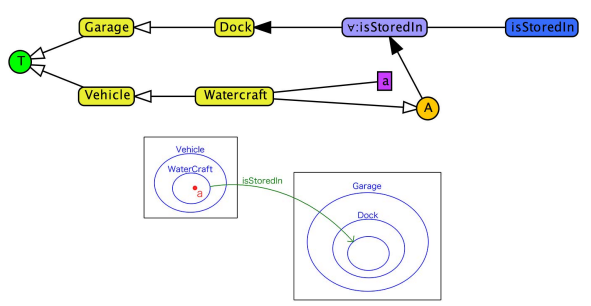

$\left(1_{2}\right)$

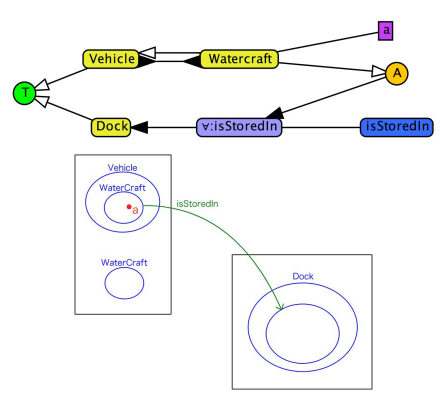

$(2)^{\prime}$

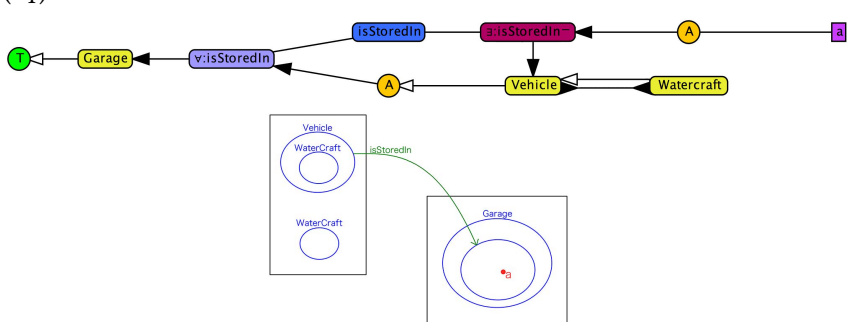

$\left.(2)_{2}\right)$

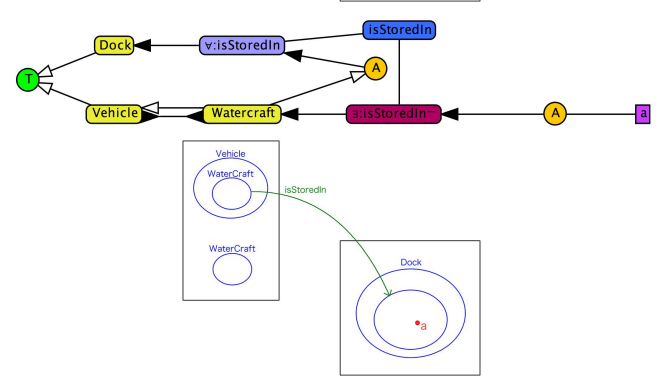

Figure 2: SOVA networks (above) and concept diagrams (below) in the four cases. $\left(1_{1}\right)$ and $\left(2_{1}\right)$ are consistent. $\left(1_{2}\right)$ and $\left(2_{2}\right)$ are contradictory. (1) can be judged without binary relations. (2) has to be judged with binary relations.

former, this diagram is contradictory. However, this diagram does not mean that there is necessarily an individual "a" which is both vehicle and watercraft. Thus, $\left(2_{1}\right)$ of Figure 2 can be judged to be consistent (not contradictory).

\subsection{Predictions}

Based on the analyses, we provide the following predictions: (i) regarding case (1) relevant to hierarchy but not to binary relations, the speed and accuracy performances of concept diagrams would be better than those of semantic networks; (ii) regarding case (2) relevant to hierarchy and binary relations, the speed and accuracy performances of concept diagrams would be better than those of semantic networks. 


\section{EXPERIMENT}

\subsection{Method}

3.1.1 Participants. Thirty-four students from the University of Brighton were recruited. The mean age was $22.53(\mathrm{SD}=5.95)$ with a range of 18-49 years. All participants gave informed consent and were paid for their participation. The School Research Ethics Panel of University of Brighton approved our experiment method. None had any prior knowledge of ontology engineering. Two participants who misunderstood the instructions were excluded. Participants were randomly divided into two groups: the SOVA network group $(\mathrm{N}=14)$ and the concept diagram group $(\mathrm{N}=18)$.

3.1.2 Materials. We presented 16 items: 8 consistent tasks and 8 inconsistent tasks (4 items in each type: see Appendix 1 for the list of tasks in OWL). The participants were presented with one diagram on a PC monitor and were asked to answer the question of whether the meaning of diagram was contradictory. The participants were asked to press the "c" button if the provided diagrams were contradictory and the " $\mathrm{n}$ " button if they were not. The tasks were presented in random order. There was no time limit. We used some fictional scenarios on superheroes and their villains, based on previous work [13].

3.1.3 Procedure. The experiment was conducted individually. First, the participants were provided with four pages of instructions on the meaning of diagrams used (see Appendix 2). Second, a pretest to check whether they understood the instructions correctly was conducted; they were presented with five diagrams of basic forms and asked to answer the questions on the existences of certain individuals in the diagrams (for the importance of pretest settings, see [24]). After the pretest, the correct answers were provided for self-checking of their answers. Fourth, the participants were provided with two pages of instruction on the meaning of (non-)contradiction, with eight examples of diagrams and explanatory sentences. Finally, the participants were provided with one task example and the general instruction. The participants were asked to respond as quickly and as accurately as possible and were instructed that they could keep the instruction sheets with them during the experiment.

\subsection{Results}

The data of the participants who made mistakes in more than two items (out of five) of the pretest were removed. In the following analysis, 3 out of 14 in SOVA network group, and 7 out of 18 participants in concept diagram group were removed, leaving 11 participants in each group.

The left of Table 1 shows the average accuracy rates of consistency checking tasks. Regarding the total 16 items, accuracy rates in the SOVA group were significantly higher than those in the concept diagram group: $80.1 \%$ for SOVA and $64.8 \%$ for concept diagrams, $t(20)=3.130, p<0.01$. Regarding the items irrelevant to binary relations, i.e., case (1), accuracy rates in the SOVA group were significantly higher than those in the concept diagram group: $83.0 \%$ for SOVA and $64.8 \%$ for concept diagrams, $t(20)=2.469, p<0.05$. Regarding the items relevant to binary relations, i.e., case (2), there was no significant difference $(t(20)=1.577): 77.3 \%$ for SOVA and $64.8 \%$ for concept diagrams.
Table 1: Accuracy rates and response times (correct answer only) in SOVA network group and concept diagram group

\begin{tabular}{lllll}
\hline \hline & \multicolumn{2}{l}{ SOVA network } & \multicolumn{2}{l}{ concept diagram } \\
\hline Case (1) : hierarchy only & $83.0 \%$ & $22.3 \mathrm{~s}$ & $64.8 \%$ & $32.2 \mathrm{~s}$ \\
Case (2) : with binary relation & $77.3 \%$ & $28.3 \mathrm{~s}$ & $64.8 \%$ & $27.7 \mathrm{~s}$ \\
\hline Total & $80.1 \%$ & $25.7 \mathrm{~s}$ & $64.8 \%$ & $30.4 \mathrm{~s}$ \\
\hline \hline
\end{tabular}

The right of Table 1 shows the response times (for correctly answered items), which were logarithmically transformed and subjected to t-tests. Regarding the total 16 items, there was no significant difference $(t(20)=0.206): 25.7 \mathrm{~s}$ for SOVA and $30.4 \mathrm{~s}$ for concept diagrams. Regarding the items irrelevant to binary relations, there was no significant difference $(t(20)=1.003): 22.3$ s for SOVA and $32.2 \mathrm{~s}$ for concept diagrams. Regarding the items relevant to binary relations, there was no significant difference $(t(20)=0.746)$ : $28.3 \mathrm{~s}$ for SOVA and $27.7 \mathrm{~s}$ for concept diagrams.

\section{GENERAL DISCUSSION}

The results of accuracy performances were contrary to our expectations in that the network-based visualization SOVA was better than the set-based visualization, concept diagrams. In particular, we predicted that in the case relevant to hierarchy but irrelevant to binary relations, distributed cognition held in comprehension of concept diagrams and thus concept diagrams were expected to be more effective than SOVA networks. Even in this case, however, SOVA networks were better than concept diagrams. These findings suggest that certain ontologies should be represented in a network-based way, rather than set-based way, in order for nonexpert users to access ontologies efficiently. The contrast between network and hierarchical-tree like visualizations in ontologies has been experimentally studied in a restricted way. Fu et al. $[5,6]$ compared the subjects' performances between networks and indented lists in extracting information of hierarchical relations. Our study extends the findings to the general scope of ontologies, in which hierarchical relations and binary relations are dealt with at the same time.

Our consideration of two contrasting visualization techniques has shed light on their use specifically for consistency checking and more general theories about visualizations. Existing theories, such as well-matchedness and well-designed representations [27] strongly suggested that concept diagrams would be superior to SOVA diagrams. By contrast, the stipulative way in which the syntax of SOVA networks gives rise to semantics would indicate that they are cognitively inferior. In our study, we surprisingly found that SOVA networks, at least for consistency checking tasks that do not require binary relations, led to superior task performance. This brings into question the importance and role of the theories of well-matchedness and well-designed representations when the goal is to devise cognitively effective visualization techniques. Thus, our study highlights the need for further cognitive theories to be developed that explain when and why one visualization technique is more effective than another - this is a key take-away message from our research. 


\section{ACKNOWLEDGMENTS}

This research was funded by a Leverhulme Trust Research Project Grant (RPG-2016-082) for the project entitled Accessible Reasoning with Diagrams.

\section{REFERENCES}

[1] Baron, M. E. 1969. A note on the historical development of logic diagrams: Leibniz, Euler and Venn. The Mathematical Gazette, 53, 113-125.

[2] Brachman, R. J.; Schmolze, J. G. 1985. An overview of the KL-ONE knowledge representation system. Cognitive Science, 9, 171-216.

[3] Corcho, O.; Roussey, C.; Blazquez, L. M. V.; Perez, I. 2009. Pattern-based OWL ontology debugging guidelines. In Proceedings of the 2009 International Conference on Ontology Patterns, CEUR vol 516 (pp. 68-82).

[4] Fekete, J.; Wang, D.; Dang, N.; Aris, A.; Plaisant, C. 2003. Overlaying graph links on treemaps. In Proceedings of 2003 IEEE Symposium on Information Visualization (pp. 82-83).

[5] Fu, B.; Noy, N. F; Storey, M. A. 2013. Indented tree or graph? A usability study of ontology visualization techniques in the context of class mapping evaluation. In Proceedings of 12th International Semantic Web Conference, Part I, LNCS 8218 (pp. 117-134). Berlin Heidelberg: Springer.

[6] Fu, B.; Noy, N. F.; Storey, M. A. 2017. Eye tracking the user experience-An evaluation of ontology visualization techniques. Semantic Web, 8, 23-41.

[7] Gennari, J. H.; Musen, M. A.; Fergerson, R. W.; Grosso, W. E.; Crubézy, M.; Eriksson, H.; Noy, N. F.; Tu, S. W. 2003. The evolution of Protégé: an environment for knowledge-based systems development. International fournal of Human-Computer Studies, 58, 89-123.

[8] Haase, P.; van Harmelen, F.; Huang, Z.; Stuckenschmidt, H.; Sure, Y. 2005. A framework for handling inconsistency in changing ontologies. In Proceedings of the 4th International Semantic Web Conference, LNCS 3729 (pp. 353-367). Berlin Heidelberg: Springer.

[9] Hammer, E. M. 1995. Logic and Visual Information. Stanford, CA: CSLI Publications.

[10] Hartley, R. T.; Barnden, J. A. 1997. Semantic networks: visualizations of knowledge. Trends in Cognitive Sciences, 1, 169-175.

[11] Holten, D. 2006. Hierarchical edge bundles: Visualization of adjacency relations in hierarchical data. IEEE Transactions on Visualization and Computer Graphics, $12,741-748$.

[12] Horridge, M.; Parsia, B.; Sattler, U. 2009. Explaining inconsistencies in OWL ontologies. In Proceedings of the 3rd International Conference on Scalable Uncertainty Management, LNAI 5785 (pp. 124-137). Berlin Heidelberg: Springer.

[13] Hou, T.; Chapman, P.; Blake, A. 2016. Antipattern comprehension: an empirical evaluation. In: Proceedings of the 9th International Conference on Formal Ontology in Information Systems (pp. 211-224). IOS Press.

[14] Howse, J.; Stapleton, G.; Taylor, K.; Chapman, P. 2011. Visualizing ontologies: A case study. In Proceedings of 10th International Semantic Web Conference, LNCS 7031 (pp. 257-272). Berlin Heidelberg: Springer.

[15] Huang, Z.; Van Harmelen, F.; Teije, A. T. 2005. Reasoning with inconsistent ontologies. In Proceedings of the 19th International foint Conference on Artificial Intelligence (pp. 454-459). Morgan Kaufmann Publishers Inc.

[16] Itzik, N.; Reinhartz-Berger, I. 2014. SOVA - A tool for semantic and ontological variability analysis. In Proceedings of the CAiSE'2014 Forum at the 26th International Conference on Advanced Information Systems Engineering, CEUR vol 1164 (pp. 177-184).

[17] Johnson, B.; Shneiderman, B. 1991. Tree-maps: A space-filling approach to the visualization of hierarchical information structures. In Proceedings of the 2nd Conference on Visualization'91 (pp. 284-291). IEEE Computer Society Press.

[18] Kalyanpur, A.; Parsia, B.; Sirin, E.; Hendler, J. 2005. Debugging unsatisfiable classes in OWL ontologies. Web Semantics: Science, Services and Agents on the World Wide Web, 3, 268-293.

[19] Lohmann, S.; Negru, S.; Haag, F.; Ertl, T. 2016. Visualizing ontologies with VOWL. Semantic Web, 7, 399-419.

[20] Mineshima, K.; Okada, M.; Takemura, R. 2012. A diagrammatic reasoning system with Euler circles. Journal of Logic, Language and Information, 21, 365-391.

[21] Mizoguchi, R.; Kozaki, K. 2009. Ontology engineering environments. In Handbook on Ontologies (pp. 315-336). Berlin Heidelberg: Springer

[22] Oliver, I.; Howse, J.; Stapleton, G.; Nuutila, E.; Törmä, S. 2009. Visualizing and specifying ontologies using diagrammatic logics. In Proceedings of the 5th Australasian Ontology Workshop, CRPIT vol 112 (pp. 37-46). Australian Computer Society, Inc.

[23] Ramakrishnan, S.,; Vijayan, A. 2014. A study on development of cognitive support features in recent ontology visualization tools. Artificial Intelligence Review, 41, 595-623.

[24] Sato, Y.; Mineshima, K. 2015. How diagrams can support syllogistic reasoning: an experimental study. Fournal of Logic, Language, and Information, 24, 409-455.
[25] Sato, Y.; Mineshima, K.; Takemura, R. 2011. Interpreting logic diagrams: A comparison of two formulations of diagrammatic representations. In Proceedings of the 33rd Annual Conference of the Cognitive Science Society (pp. 2182-2187). Austin, TX: Cognitive Science Society.

[26] Stapleton, G.; Howse, J.; Chapman, P.; Delaney, A.; Burton, J.; Oliver, I. 2013. Formalizing concept diagrams. In Proceedings of the 9th International Conference on Distributed Multimedia Systems (pp. 182-187). Skokie, IL: Knowledge Systems Institute.

[27] Zhang, J.; Norman, D. A. 1994. Representations in distributed cognitive tasks. Cognitive Science, 18, 87-122.

[28] Zhao, S.; McGuffin, M. J.; Chignell, M. H. 2005. Elastic hierarchies: Combining treemaps and node-link diagrams. In Proceedings of 2005 IEEE Symposium on Information Visualization (pp. 57-64).

Appendix 1: A list of OWL expressions of tasks

https://sites.google.com/site/myardproject/exp/ListTasks.zip?attredirects=0\&d=1

Appendix 2: Materials and instructions used in the experiment https://sites.google.com/site/myardproject/exp/MateInst.zip?attredirects=0\&d=1 